\title{
Interplay of transcription factors and microRNAs during embryonic hematopoiesis
}

\author{
Xueping Gong ${ }^{\dagger}$, Ruihua Chao ${ }^{\dagger}$, Pengxiang Wang, Xiaoli Huang, Jingjing Zhang, Xiaozhou Zhu, \\ Yanyang Zhang, Xue Yang, Chao Hou, Xiangjun Ji, Tieliu Shi \& Yuan Wang* \\ Shanghai Key Laboratory of Regulatory Biology, Institute of Biomedical Sciences and School of Life Sciences, East China Normal University, \\ Shanghai 200241, China
}

Received June 11,2016; accepted August 10,2016; published online October 17, 2016

\begin{abstract}
Hematopoietic stem cells (HSCs), which are localized in the bone marrow of adult mammals, come from hematopoietic endothelium during embryonic stages. Although the basic processes of HSC generation and differentiation have been described in the past, the epigenetic regulation of embryonic hematopoiesis remains to be fully described. Here, by utilizing an in vitro differentiation system of mouse embryonic stem cells (ESCs), we identified more than 20 microRNAs that were highly enriched in embryonic hematopoietic cells, including some (e.g. miR-10b, miR-15b, and miR-27a) with previously unknown functions in blood formation. Luciferase and gene expression assays further revealed combinational binding and regulation of these microRNAs by key transcription factors in blood cells. Finally, bioinformatics and functional analyses supported an interactive regulatory control between transcription factors and microRNAs in hematopoiesis.
\end{abstract}

hematopoiesis, embryonic stem cells, microRNA

Citation: $\quad$ Gong, X., Chao, R., Wang, P., Huang, X., Zhang, J., Zhu, X., Zhang, Y., Yang, X., Hou, C., Ji, X., Shi, T., and Wang, Y. (2017). Interplay of transcription factors and microRNAs during embryonic hematopoiesis. Sci China Life Sci 60, 168-177. doi: 10.1007/s11427-016-0168-0

\section{INTRODUCTION}

Hematopoiesis is a complex biological process in which blood cells are continuously generated from hematopoietic stem cells (HSCs) (Godin and Cumano, 2002). In general, mammalian embryonic hematopoiesis occurs in two major waves. For example, in mice, primitive blood cells emerge in yolk sac around day 7.5 before birth, whereas definitive HSCs appear in the region of aorta-gonad-mesonephros 1-2 days later (Bertrand et al., 2005; Cumano et al., 2000; Dzierzak, 1999; Lensch and Daley, 2004; Long and Huang, 2015; Medvinsky and Dzierzak, 1996; Palis et al., 1999; Zhao and $\mathrm{Li}, 2015$ ). This dynamic developmental program

$\dagger$ Contributed equally to this work

*Corresponding author (email: ywang@bio.ecnu.edu.cn) requires exact orchestration of intrinsic and extrinsic signals to control HSC proliferation, differentiation, and survival (Godin and Cumano, 2002). Multiple master transcription factors are vital determinants in this regulatory machinery and tight coordination is needed (Elefanty et al., 1997; Mankertz et al., 2004; Morrison et al., 1995; Schmitt et al., 1991). Although genome-wide ChIP-Seq analyses implicated a combinational interaction between a heptad of transcription factors including SCL, LYL1, LMO2, GATA2, RUNX1, ERG, and FLI1 in adult HSCs (Wilson et al., 2010), their interplay to regulate embryonic hematopoiesis is awaiting for further discovery.

The fine balance between HSC self-renewal and lineage specification is also stringently regulated by interplays between genetic and epigenetic modifiers. Among the epigenetic regulators, microRNAs (miRNAs) are a class of small 
22 nt non-coding RNAs that mainly mediate post-transcriptional control of gene expression via transcript degradation or by inhibiting protein translation (Bartel, 2004; Gangaraju and Lin, 2009). Although there is clear evidence supporting miRNAs as important players for hematopoietic development and leukemic formation (Garzon and Croce, 2008; Vasilatou et al., 2010), potential interactions between key transcription factors and miRNAs in embryonic hematopoiesis are yet to be understood.

The number of HSCs is very limited in both embryo and in adult bone marrow (BM) (Morrison et al., 1995). As such, in vitro differentiation of embryonic stem cells (ESCs) provides an alternative resource for the study of regulatory events in hematopoiesis (Keller et al., 1993; Kyba and Daley, 2003; Xie and Zhang, 2015; Xie et al., 2014). During ESC differentiation, multi-potential FLK $1^{+}$hemangioblasts developed from Brachyury ${ }^{+}$mesodermal population (Kennedy et al., 1997; Kennedy et al., 2007), followed by the formation of $\mathrm{CD} 1^{+}$primitive HSCs (McKinney-Freeman et al., 2008; Mikkola et al., 2002) and $\mathrm{CD}^{+} 5^{+}$mono-nucleated blood cells (Hermiston et al., 2003). In this study, we analyzed the expression of miRNAs during mouse ESC differentiation through expression arrays, and identified multiple novel miRNAs highly enriched in $\mathrm{CD} 41^{+}$and $\mathrm{CD} 45^{+}$cells. Further analyses demonstrated that these hematopoiesis-related miRNAs were regulated by a combinational interaction of key transcriptional factors in blood cells.

\section{RESULTS}

\section{The expression of miRNAs during embryonic hematopoiesis}

Previous studies demonstrated that ESCs could differentiate into three-dimensional structures called embryoid bodies (EBs) and mimic primitive hematopoietic development in vitro (Keller et al., 1993; Kyba and Daley, 2003; Wang et al., 2005). To understand the role of miRNAs in this process, we utilized the in vitro differentiation system of mouse ESCs with a GFP transgene driven by the Brachyury $(\mathrm{T})$ promoter. $\mathrm{T}_{-\mathrm{GFP}^{+}}$(mesodermal population), $\mathrm{FLK}^{+}$(multi-potential hemangioblasts), $\mathrm{CD} 1^{+}$(hematopoietic precursors), and $\mathrm{CD} 45^{+}$cells (mononuclear blood cells) were isolated at various time points along EB differentiation (Figure 1A), and microarray analyses were performed. More than 200 miRNAs demonstrated differential expression levels in these sorted populations. Among them, several hematopoiesis-related miRNAs (including miR-451, miR-142, and miR-146a/b, etc. (Chen et al., 2004; Fatica et al., 2006; Garzon and Croce, 2008; Vasilatou et al., 2010)) were highly enriched in $\mathrm{CD}_{4} 1^{+}$and/or $\mathrm{CD}_{4} 5^{+}$cells (Figure 1B). By contrast, miRNAs implicated in maintaining pluripotency, such as miR-302, miR-293/295, and miR-363, etc. (Gruber et al., 2014; Houbaviy et al., 2003; Judson et al., 2009; Wang et al.,
2008), were significantly down regulated in those isolated blood cells from ESCs (Figure 1B). Importantly, we found increased levels of several miRNAs such as miR-10b and miR-15a in $\mathrm{CD}_{4} 1^{+}$or $\mathrm{CD} 45^{+}$cells (Figure 1B). Roles of these miRNAs in hematopoiesis are unknown but our data suggest their potential functions in blood formation.

To confirm these results, we further utilized another ESC line, Ainv15, and compared the expression of these miRNAs from sorted $\mathrm{CD} 41^{+}$or $\mathrm{CD} 45^{+}$cells with $\mathrm{CD} 41^{-}$or $\mathrm{CD} 45^{-}$populations by real-time RT-PCR analyses. Indeed, the miRNAs that demonstrated an increased expression in blood cells compared to $\mathrm{T}_{-} \mathrm{GFP}^{+}$mesodermal precursors were enriched in the $\mathrm{CD} 41^{+}$or/and $\mathrm{CD} 45^{+}$populations (Figure $1 \mathrm{C}$ and D), whereas miRNAs that were highly expressed in ESCs showed decreased levels in $\mathrm{CD}_{4} 1^{+}$and/or $\mathrm{CD} 45^{+}$cells compared to their negative controls (Figure 1C and D), demonstrating a high quality of the microarray data.

\section{Promoters of miRNAs are bound by multiple key tran- scription factors in hematopoiesis}

We next explored the upstream regulators of these miRNAs enriched in blood cells with promoter analyses (Figure 2A). Among the transcription factors we examined, GATA3 and SCL were master regulators involved in both primitive and definitive hematopoiesis (Chen and Zhang, 2001; Gao et al., 2016; Porcher et al., 1996), whereas EKLF (or KLF1) and PU.1 participated in erythropoiesis and lymphogenesis respectively (Lohmann and Bieker, 2008; Nerlov and Graf, 1998). Interestingly, binding motifs of multiple transcription factors including EKLF and PU.1 that participated in those different processes of hematopoiesis were identified at adjacent motifs within the same promoter regions of miR-144/451, miR-223, and miR-142 (Figure 2A). To confirm these results, Chromatin Immuno Precipitation (ChIP)-PCR assays were performed in MEL (a mouse erythroleukemia line) with enforced expression of FLAG-tagged GATA3, EKLF, and PU.1. Indeed, all three transcription factors were bound at the predicated regions in promoters of target miRNAs (Figure 2B). For example, although miRNA-144 was believed to play a role in erythrocyte formation, several binding motifs for GATA3, EKLF, and PU.1 were found to be co-localized at 400-500 bp upstream of transcription start site (TSS) for miRNA-144 (Figure 2A and B). These results thus suggest that EKLF and PU.1 may cooperate to fine-tune the transcript level of miRNA-144 in erythrocytes and myeloid/lymphoid lineages. Taken together, our data demonstrate a combinational cooperation of transcription factors for regulating the expression of miRNAs involved in hematopoiesis.

\section{Expression of multiple miRNAs is regulated by key tran- scription factors in hematopoiesis}

We next examined whether these key transcription factors 

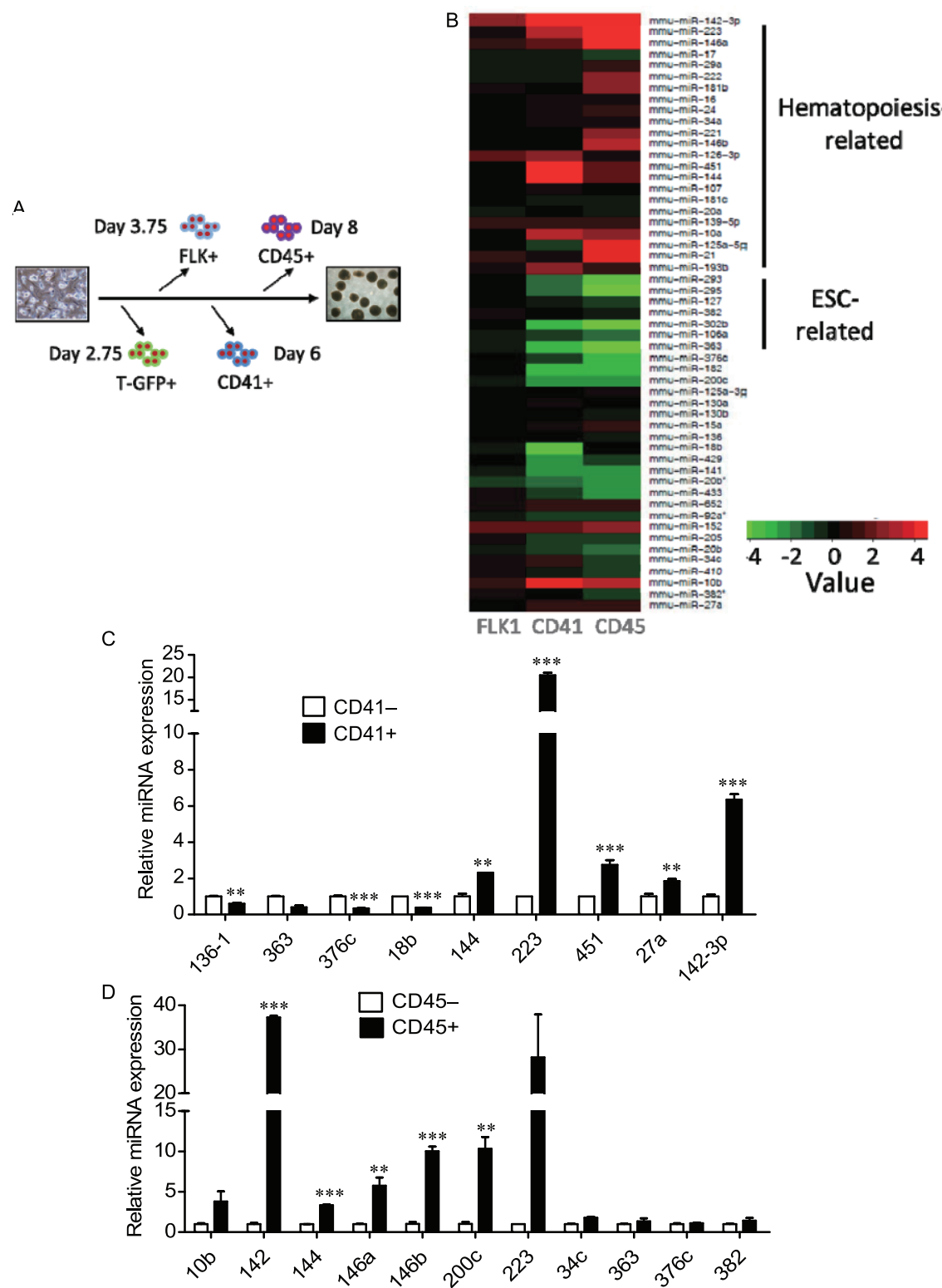

Figure 1 Identify miRNAs that are upregulated during embryonic hematopoiesis. A, A work flow to isolate embryonic blood cells along ESC differentiation. B, Heatmap of a set of miRNAs that were differentially expressed at distinct stages of blood formation. C and D, Real-time RT-PCR analyses of miRNAs in sorted $\mathrm{CD} 41^{-}$and $\mathrm{CD}_{4} 1^{+}(\mathrm{C})$ or in $\mathrm{CD} 45^{-}$and $\mathrm{CD} 45^{+}$(D) blood cells derived from differentiated ESCs. C and D, Relative expression levels of miRNAs in $\mathrm{CD} 41^{+}$to $\mathrm{CD} 41^{-}$or in $\mathrm{CD} 45^{+}$to $\mathrm{CD} 45^{-}$cells are represented as mean $\pm \mathrm{SE}$ of replicates from multiple representative experiments. $*, P<0.05 ; * *, P<0.01 ; * * *$, $P<0.001$.

indeed regulated the expression of their target miRNAs by a firefly and renilla dual luciferase reporter assay. In this assay, firefly luciferase driven by promoters of miRNAs was co-transfected with expressing plasmids encoding renilla luciferase and FLAG-tagged transcription factors into 293T cells, and relative firefly (to renilla) luciferase activity was measured at $24-48 \mathrm{~h}$ post transfection. Consistent with the prediction and ChIP-PCR data above, the master regulator, GATA3, demonstrated the strongest upregulation of all miRNAs we tested, whereas EKLF and PU.1 exhibited a differential modulation of miRNA expression in this assay (Figure 3A). In addition, GFI1b mainly upregulated the expression of miR-142 (Figure 3A). By contrast, overexpression of AML1, a protein involved in both self-renewal of hematopoietic stem cells and myeloid-lymphoid formation, decreased the transcript levels of all four miRNAs (Figure $3 \mathrm{~A})$, suggesting that a general inhibitory co-factor for AML1 may exist in $293 \mathrm{~T}$ cells.

We further examined the transcript levels of the miRNA precursors (pri-miRNAs) by real-time RT-PCRs in MEL cells upon enforced expression of these transcription factors. Indeed, GATA3 showed a dramatic upregulation of miR-144 and miR-223, while EKLF mainly increased the expression of both miR-144/451 and miR-142 (Figure 3B). In addition, 

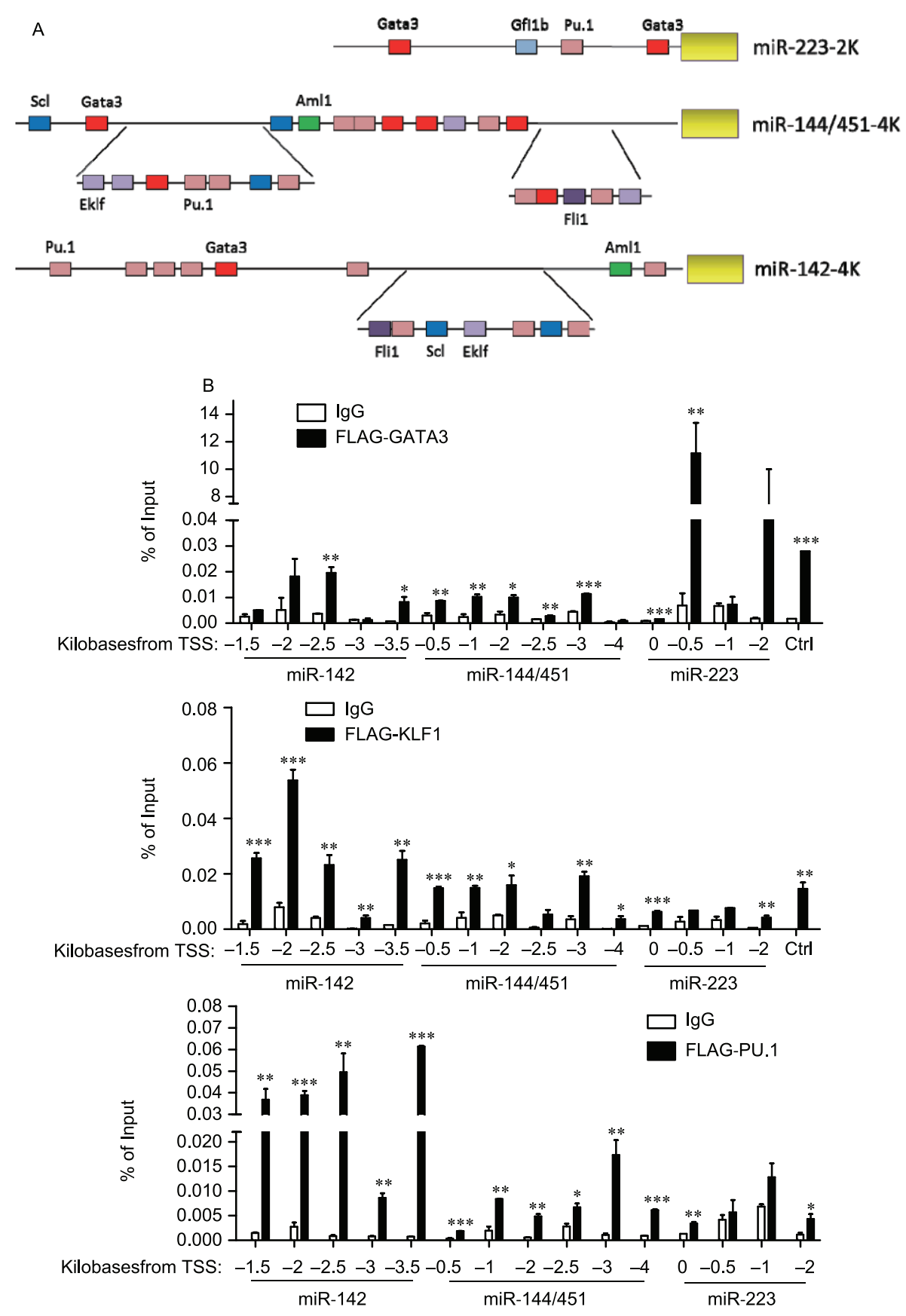

Figure 2 The promoters of miRNAs were co-bound by multiple transcription factors. A, Predication of binding sites for transcription factors on the promoters of miRNAs. B, ChIP-PCR analyses with a FLAG antibody against FLAG-tagged transcription factors on genomic DNA extracted from MEL. TSS, transcription start site. Negative numbers below panels indicate the upstream distance from TSS of target miRNAs. Relative percentage of input pulled down by the FLAG antibody to a control $\mathrm{IgG}$ was represented as mean $\pm \mathrm{SE}$ of replicates from multiple representative experiments. $*, P<0.05 ; * *, P<0.01 ; * * *, P<0.001$.

ETS family members including FLI1 and ERG upregulated miR-142 and miR-223, but decreased the level of miR-144/451 pri-miRNAs (Figure 3B). Notably, the regulatory roles of these factors were not exactly the same as those observed in 293 T cells. For example, ectopic AML1 expression displayed an inhibition of all four miRNAs that we examined in $293 \mathrm{~T}$ cells, but this suppression was not observed in MEL (Figure 3B). By contrast, AML upregulated the transcript level of miRNA-223 (Figure 3B). These result support the importance of setting and specificity of blood cells (eg. MEL) in which transcriptional regulators are needed to perform their biological functions.

To further understand the upstream regulators of miRNAs during hematopoiesis in vivo, we established multiple conditional ESC lines in which the expression of key transcriptional factors was induced upon treatment of doxycycline (Figure 4A-D). In this assay, we found that SCL and EKLF, the genes that were reported to be involved in eryth- 
A
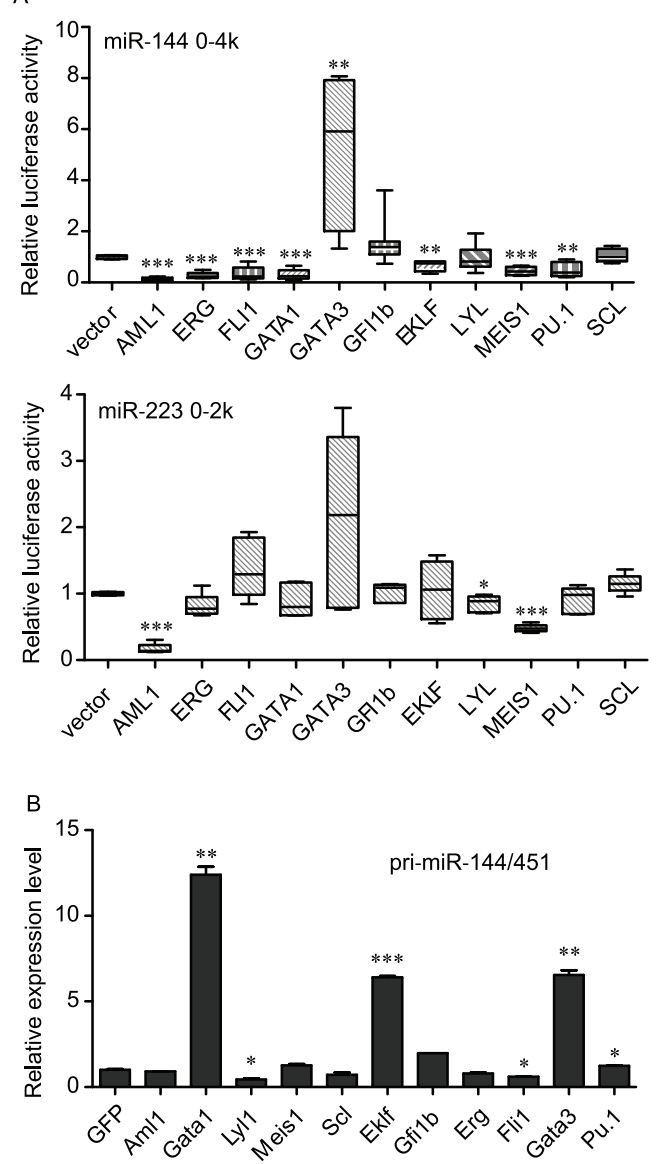
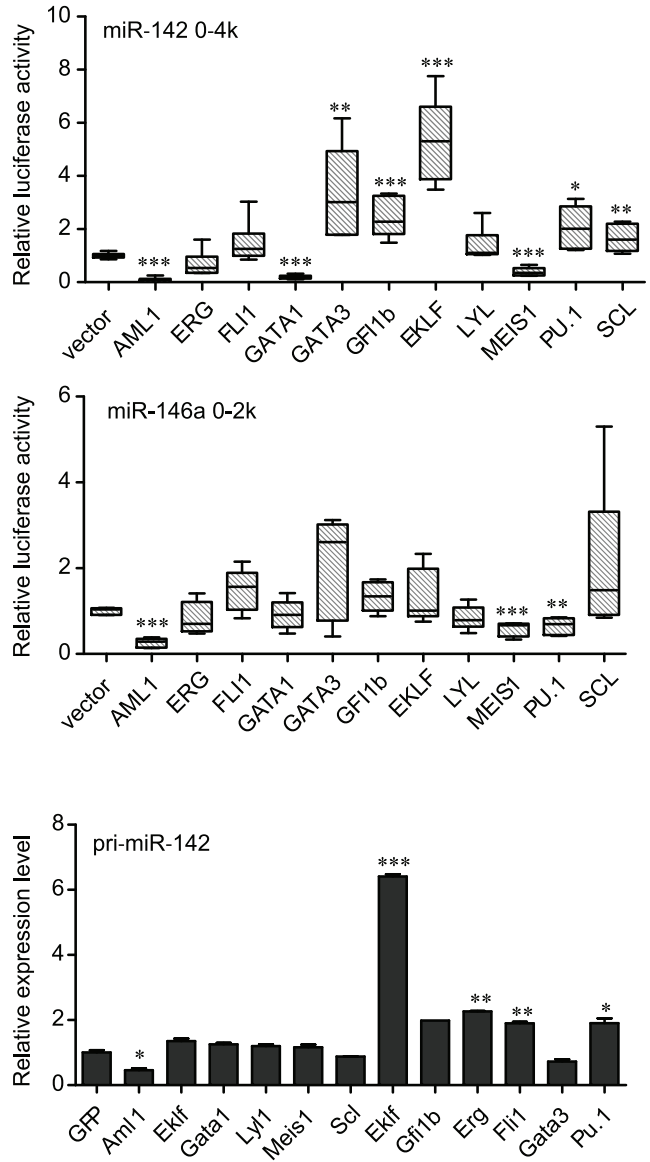

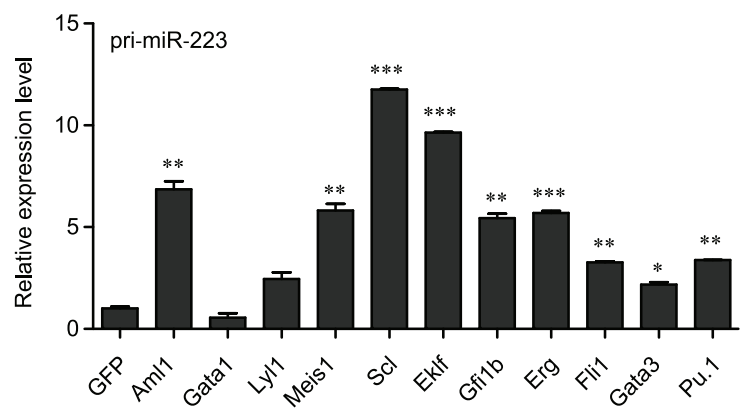

Figure 3 The expression of miRNAs were regulated by combinational control of multiple transcription factors. A, Luciferase activities driven by upstream promoter regions of miRNAs were examined upon co-transfection of luciferase reporter plasmids and transgenes to express transcription factors or an empty vector control. Relative firefly luciferase activity to the Renilla transfection control is represented as mean \pm SE of replicates from at least three independent experiments. B, Relative pri-miRNA levels upon enforced expression of various transcription factors in MEL cells were examined by real-time RT-PCR assays. Data were represented as mean $\pm \mathrm{SE}$ of replicates from at least three independent experiments. $*, P<0.05 ; * *, P<0.01 ; * * *, P<0.001$.

ropoiesis mainly upregulated miR-144/451 and miR-142, whereas FLI1 and ERG, the key factors in megakaryocyte formation (Kruse et al., 2009), significantly increased the expression of miR-142 and miR-146b (Figure 4A-D), implicating distinct roles of their target miRNAs during primitive hematopoiesis.

\section{Differential effects of microRNAs on hematopoiesis dur- ing ESC differentiation}

To explore the functions of miRNAs during embryonic hematopoiesis, we established conditional miRNA ESC lines, in which the expression of miRNAs was enforced by doxycycline treatment (Figure 5A). We previously reported that $\mathrm{CD} 71^{\text {high }}$ population derived from ESCs represents primitive erythroid precursors (Chao et al., 2014), whereas CD45 marked differentiated mononuclear blood cells (Hermiston et al., 2003), with CD41 as a marker for primitive HSCs (McKinney-Freeman et al., 2008; Mikkola et al., 2002). During ESC in vitro differentiation, we found that both miR-142 and miR-146b transiently increased the formation of $\mathrm{CD} 71^{\text {high }}$ population (Figure $5 \mathrm{~B}$ ), indicating previously unknown functions of these miRNAs in primitive erythro- 

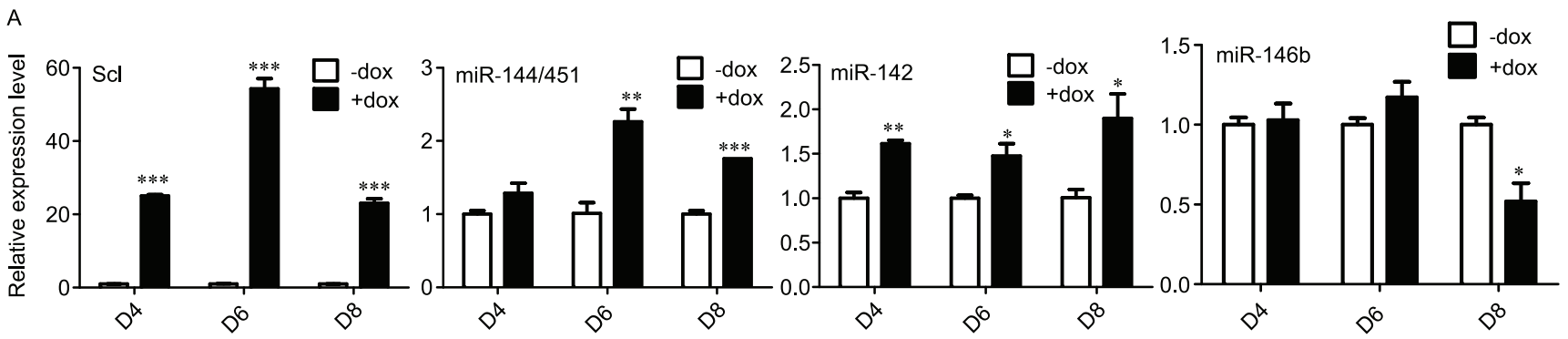

B
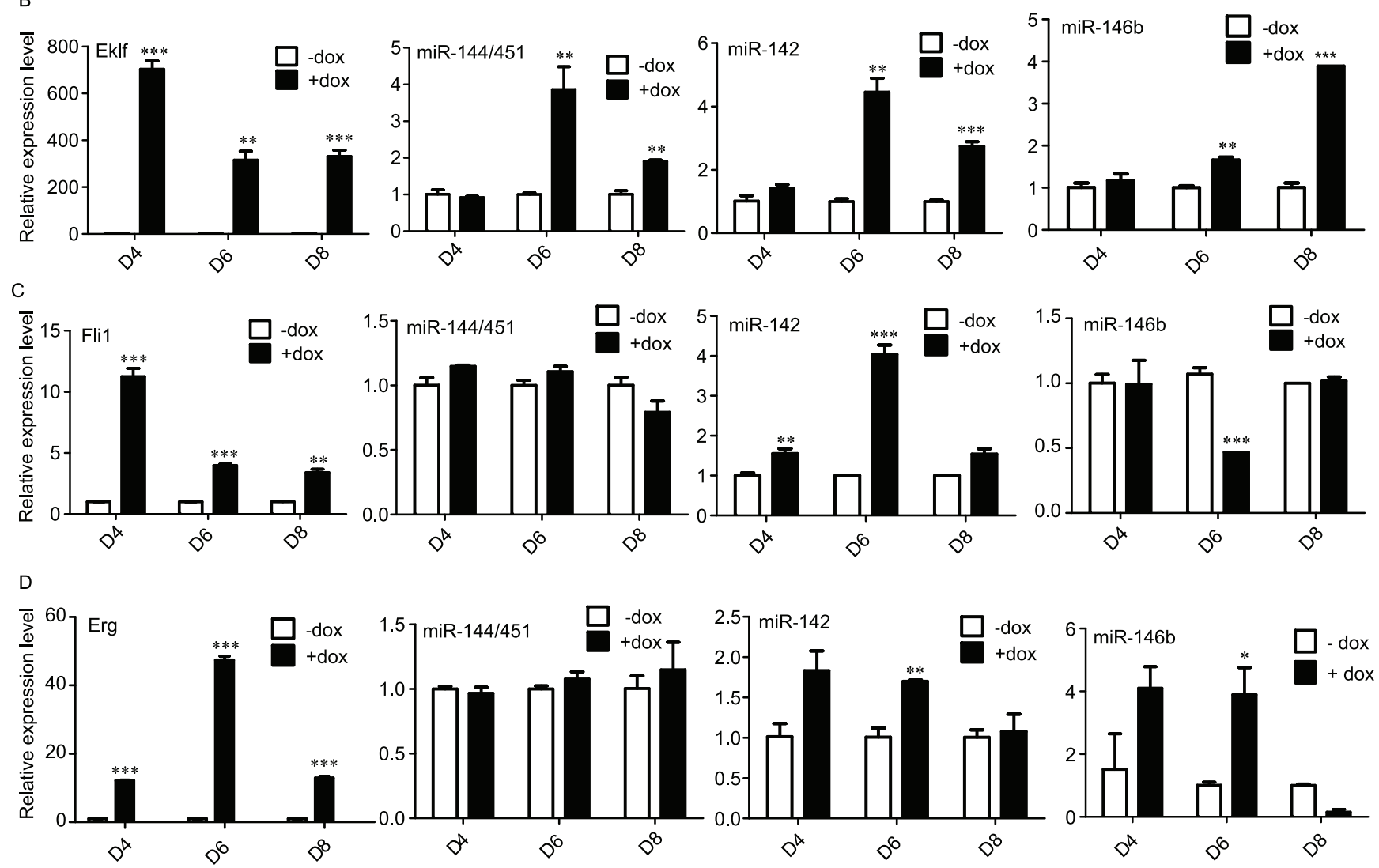

Figure 4 The expression of miRNAs was regulated by various transcription factors during ESC differentiation. A-D, The inducible ESC lines were established to enforce the expression of various transcription factors in the presence of doxycycline (Dox). The overexpression of targeted transcription factors were confirmed by RT-PCR analyses (left panels in A-D). Relative expression levels of pri-miRNAs were measured by real-time RT-PCR assays in whole EB population at various time points during ESC differentiation. - or + dox: upon induction of expression of transcription factors with $(+)$ or without $(-)$ doxycycline (Dox) treatment. Data are represented as mean $\pm \mathrm{SE}$ of replicates from multiple representative experiments. $*, P<0.05 ; * *, P<0.01 ; * * *, P<0.001$.

poiesis. In addition, the percentage of $\mathrm{CD} 41^{+}$cells was upregulated upon induction of miR-146b (Figure 5B), suggesting a role of this miRNA in primitive hematopoiesis. Surprisingly, there was no obvious alteration in formation of CD71 ${ }^{\text {high }}$ population upon enforced expression of miR-144/451, a previously reported miRNA in the erythroid formation (Patrick et al., 2010). It is possible that sole overexpression of miR$144 / 451$ was not sufficient to induce primitive erythroid fate specification during ESC differentiation.

Interestingly, although these miRNAs participate in hematopoiesis, many of their potential target genes are the same key transcription factors that regulate miRNA expression in blood development (Figure 6A). For example, bioinformatics prediction indicated that miR-144/451 targeted 3'-UTR of FLII and ERG, which in turn appeared to inhibit the expression of miR-144/451, and thus formed a negative interactive feedback loop (Figure 6). In addition, our data suggested GATA3 and SCL activated the expression of miR-144/451 in HSCs, in turn miR144 might target GATA3 and SCL (via bioinformatics prediction) to facilitate erythrocyte fate specification at later stages of hematopoiesis. Similarly, whereas EKLF upregulated miR-223 level, miR-223 likely targeted EKLF to negatively regulate erythrocyte formation in myeloid lineages (Figure 6). Taken together, these data demonstrated that key transcription factors and miRNAs interact with each other to fine-tune their expression and function during hematopoietic development. 

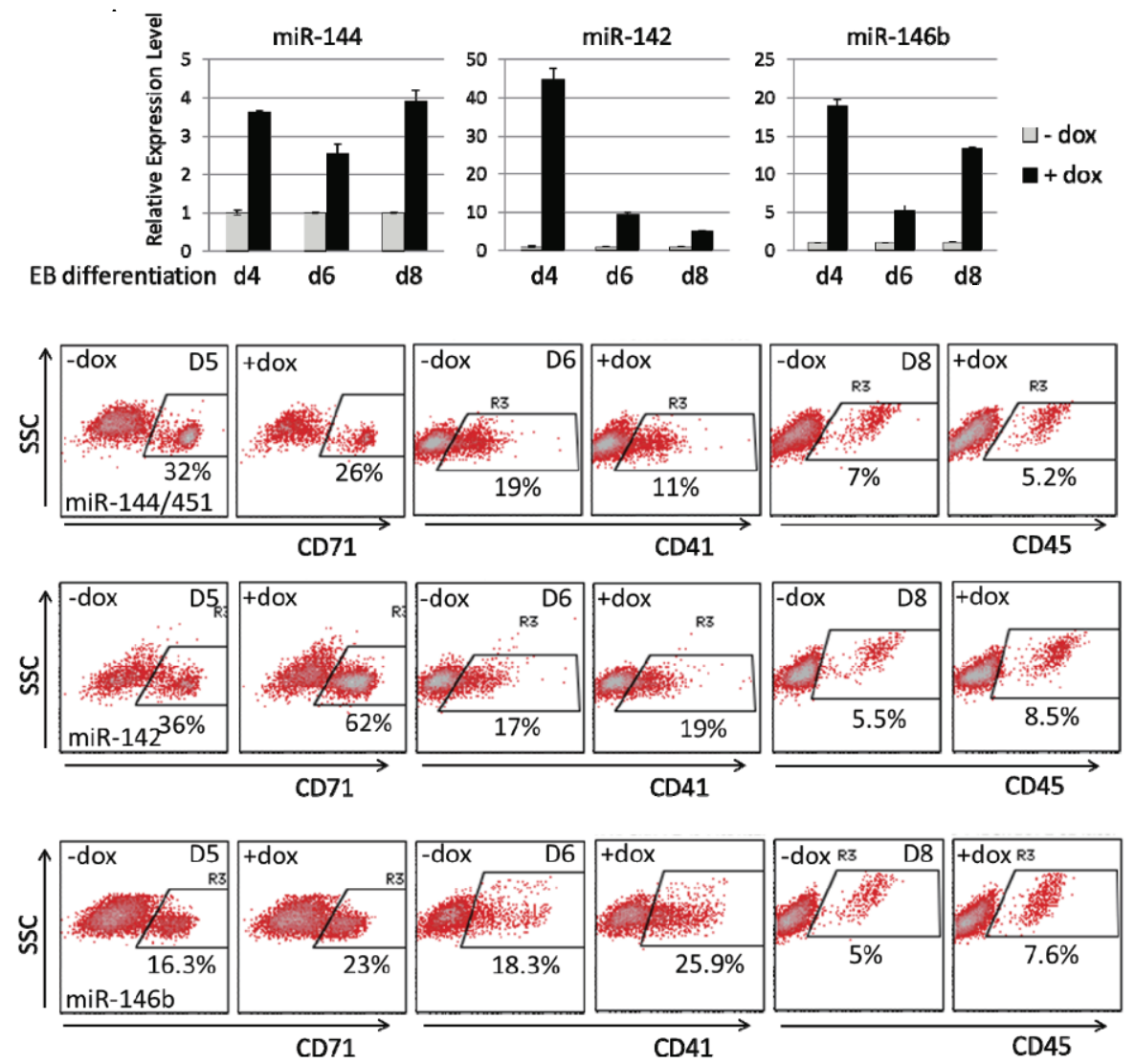

Figure 5 (Color online) The differential influence of miRNAs on primitive hematopoiesis during ESC differentiation. A, The conditional ESC lines were established to enforce the inducible expression of various pr-miRNAs in the presence of doxycycline. The overexpression of targeted pri-miRNAs were confirmed by quantitative RT-PCR analyses of whole EB population at various time points during ESC differentiation. B, The percentage of primitive erythroblasts $\left(\mathrm{CD} 71^{\text {high }}\right)$, primitive hematopoietic stem cells $\left(\mathrm{CD} 41^{+}\right)$, and mononuclear blood cells $\left(\mathrm{CD} 45^{+}\right)$were measured by flow cytometry on whole EB population at various time points along ESC differentiation. This experiment was conducted multiple times with similar results.

\section{DISCUSSION}

Although many miRNAs have been demonstrated to be important during definitive hematopoiesis and leukemic formation in adulthood, roles of these miRNAs in embryonic primitive blood development are yet to be understood. In this study, we identified more than 20 miRNAs that were highly enriched in primitive hematopoiesis from mouse ESCs, including the ones that had not been previously reported (e.g. miR-10b). These data thus support the notion that ESC in vitro differentiation system is a powerful platform to identify novel regulators during embryonic development.

To gain knowledge in transcriptional regulation is key to understand the mechanism that governs complex biological processes such as hematopoiesis. Previous studies demonstrated that transcriptional factors at the same stage of adult hematopoiesis cooperated to regulate their downstream targets (Wilson et al., 2010). Our bioinformatics prediction and ChIP-PCR analyses suggest that multiple key transcription factors from distinct steps of hematopoiesis are also co-localized at adjacent positions at promoters of their targeted miRNAs. This observation reveals a combinational coordi- nation and functional relevance among these transcriptional regulators. Alternatively, these miRNAs may play multiple roles at distinct stages of hematopoietic development. In addition, although reporter assays in $293 \mathrm{~T}$ cells indicated an upregulation of luciferase activity driven by the promoter of miR-142 upon GATA3 overexpression, the level of miR-142 was not changed by increased level of GATA3 in MEL, an erythroleukemic line (Figure 3). Similarly, SCL enhanced the expression of miR-223 only in hematopoietic population (Figure 3), suggesting that additional factors in blood cells may be needed in coordination with GATA3 or SCL to modulate the expression of their targeted miRNAs.

It was previously demonstrated that SCL and EKLF promoted erythropoiesis, whereas ETS family members, such as ERG and FLI1, enhanced megakaryopoietic differentiation by inhibiting erythrocyte formation (Kruse et al., 2009; Starck et al., 2010). We found that both SCL and EKLF increased the expression of miR-144/451, whereas FLI1 downregulated miR-144/451, in turn miR-144/451 might target FLI1 to inhibit its expression in erythrocytes, thus forming a negative feedback circuitry to fine-tune the formation of red blood cells. Similarly, miR-223, a miRNA implicated in 

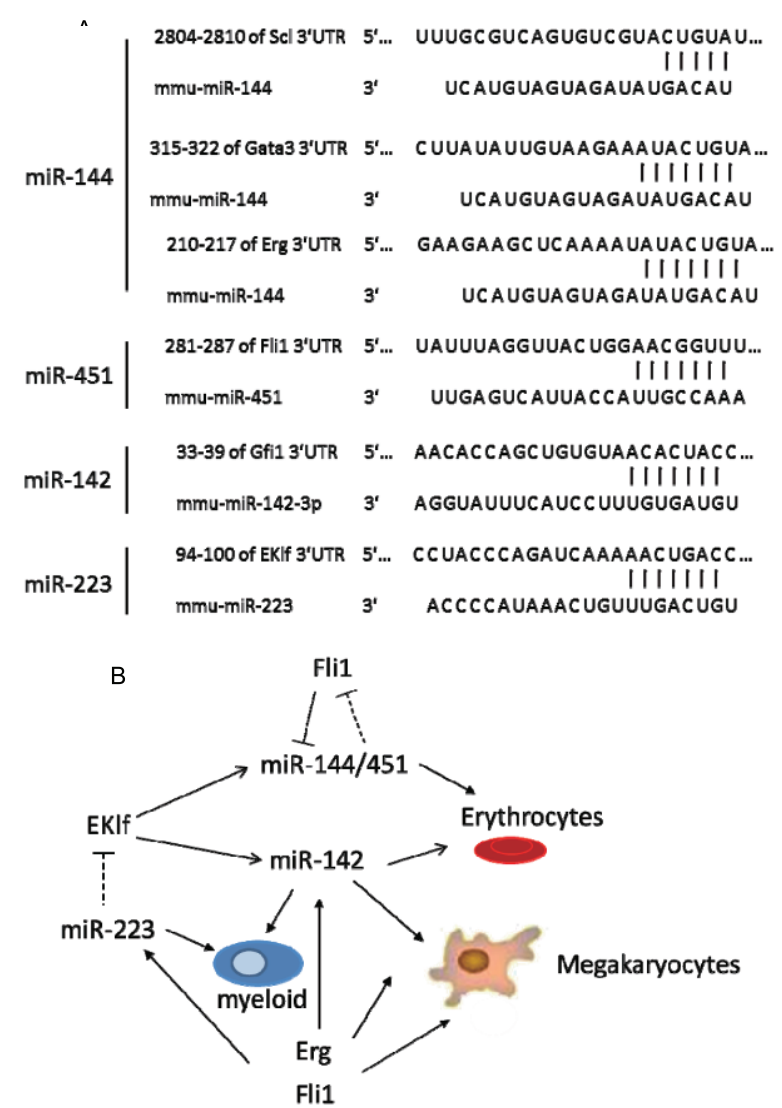

Figure 6 (Color online) The interacting loop of miRNAs and transcription factors. A, Predication of target genes regulated by miRNAs. B, The interacting loop of miRNAs and transcription factors identified in this study was summarized.

myeloid-lymphocyte formation (Fazi et al., 2005), might target 3'-UTR of EKLF and repress it expression in myeloid cells. Interestingly, ETS family members, SCL, and EKLF all enhanced the expression of miR-142, suggesting its role in both erythropoietic and megakaryopoietic development. Indeed, we observed that enforced expression of miR-142 or miR-146b transiently increased the formation of $\mathrm{CD} 71^{\text {high }}$ population from ESCs, indicating previously unknown roles of these miRNAs in primitive erythropoiesis. Taken together, our data demonstrated a combinational interplay between key transcription factors and miRNAs during embryonic hematopoiesis.

\section{MATERIALS AND METHODS}

\section{Plasmid construction}

Mouse AML1, ERG, FLI1, GATA1, GATA3, GFI1B, LYL1, MEIS1, PU.1 and SCL were amplified from a cDNA library of mouse differentiated ESCs and cloned in fusion with a $1 \times$ FLAG tag into plvx-IRES-zsgreen for luciferase reporter assay, or into pll3.7-IRES-GFP for retroviral infections of MEL cells. Similarly, ERG, EKLF and FLII cDNAs were cloned into plox for inducible expression of these transcription factors. For construction of inducible expression of primiRNAs, about 300 bp upstream and downstream of flanking regions of mature miRNAs were amplified from mouse genomic DNA and cloned into plox plasmid. Primers for cloning in this study are listed in Table S1 in Supporting Information.

\section{ESC cell culture and differentiation}

Mouse ESCs were cultured on irradiated mouse embryonic fibroblasts (MEFs) in high glucose Dulbecco's modified eagle medium (DMEM) supplemented with $15 \%$ fetal bovine serum (FBS), $2 \mathrm{mmol} \mathrm{L}^{-1} \mathrm{~L}$-glutamine, $100 \mathrm{U} \mathrm{mL}^{-1}$ penicillin and streptomycin (all from Gibco-BRL, USA), $1000 \mathrm{U} \mathrm{mL}^{-1}$ LIF (Millipore, USA), $100 \mu \mathrm{mol} \mathrm{L}{ }^{-1} \beta$-mercaptoethanol, and $1 \%$ nonessential amino acids (NEAA) (Gibco-BRL). For differentiation, ESCs were trypsinized into single cells, collected in EB differentiation medium (IMDM/15\% FBS, 200

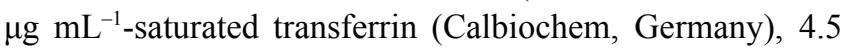
mmol L ${ }^{-1}$ monothiolglycerol and $50 \mu \mathrm{g} \mathrm{mL}^{-1}$ ascorbic acid (Sigma, USA), and $2 \mathrm{mmol} \mathrm{L}^{-1} \mathrm{~L}$-glutamine, $100 \mathrm{U} \mathrm{mL}^{-1}$ penicillin and streptomycin, all others from Gibco-BRL), and plated for $45 \mathrm{~min}$ to allow MEFs to adhere. Non-adherent ESCs were collected and plated in hanging drops at 500 cells per $15 \mu \mathrm{L}$ drop in an inverted bacterial petri dish. Embryoid bodies (EBs) were collected at day 2 and transferred into $10 \mathrm{~mL}$ EB differentiation medium in slowly rotating $10 \mathrm{~cm}$ petri dishes. Cells were harvested at different time points by collagenase treatment. To induce gene expression, $0.5-1$ $\mu \mathrm{g} \mathrm{mL} \mathrm{m}^{-1}$ doxycycline was added to the culture medium from day 2. Stable ESC lines were established by selection of hygromycine resistant ESCs after electroporation. MEL cells were cultured in RPMI-1640 medium supplemented with $10 \% \mathrm{FBS}, 100 \mu \mathrm{g} \mathrm{mL} \mathrm{mL}^{-1}$ streptomycin, and $100 \mathrm{U} \mathrm{mL}^{-1}$ penicillin.

\section{Generation of the doxycycline-inducible ESC lines}

The protocol to generate doxycycline inducible ESC lines was published previously (Kyba et al., 2002; Wang et al., 2005). In brief, the parental ESC line Ainv15, was electroporated with $20 \mu \mathrm{g}$ plox-ERG, plox-EKLF, or plox-FLI1, together with $20 \mu \mathrm{g}$ the Cre recombinase expression plasmid, pSalk-Cre, followed by selection in ESC culture medium with $150 \mu \mathrm{g} \mathrm{mL} \mathrm{mL}^{-1}$ hygromycine (GIBCO). Individual colonies were picked at day 12 post drug treatment for further expansion. The ESC line with inducible SCL expression is a kind gift from Dr. George Daley's lab at Harvard Medical School.

\section{Flow cytometry analysis and cell sorting}

Dissociated EB cells were incubated with appropriate primary antibodies for $30 \mathrm{~min}$ at $4^{\circ} \mathrm{C}$, washed twice, and analyzed with FACSCalibur (BD Biosciences, USA) for analy- 
ses or sorted with FACSAria II (BD Biosciences). All monoclonal antibodies used in this study were purchased in fluorochrome-coupled forms from BD Pharmingen.

\section{Total RNA preparation, RT-PCR, and quantitative real- time PCR}

Total RNAs were extracted with Trizol (Invitrogen, USA) and cDNAs were synthesized using a PrimeScript ${ }^{\mathbb{R}}$ RT reagent Kit (TaKaRa, Japan). Real-time PCR was performed on a Stratagene MX3000P instrument and analyzed as described previously (Wang et al., 2013). Primers used in this study are listed in Table S1 in Supporting Information.

\section{Chromatin immunoprecipitation}

Cells were cross-linked with $1 \%$ formaldehyde and sonicated into $200 \mathrm{bp}$ fragments. The cell lysate was precleared before being immunoprecipitated with a FLAG antibody (Sigma) or control IgG. Followed by a pull-down with protein $\mathrm{A} / \mathrm{G}$ beads (Santa Cruz Biotechnology, USA), a subsequent series of washes, and elution, enrichment of targeted DNA fragments was finally examined by real-time RT-PCR. Primers for ChIP-PCR used in this study are listed in Supplementary Table 1.

\section{Luciferase reporter assay}

The upstream DNA fragments of mouse miR-144/451, miR223 , and miR142 were amplified by PCR and cloned into pGL4-basic firefly luciferase reporter vector. The reporter plasmid was then co-transfected into 293T cells with a Renilla-luciferase expressing vector, and plvx-ires-zsgreen plasmid containing transgenes of transcription factor driven by a CMV promoter. The activity of firefly relatively to renilla luciferase was measured with a luciferase assay kit (Promega, USA).

\section{Microarray assay}

The $\mathrm{GFP}^{+}$(Brachyury-GFP), $\mathrm{Flk1}^{+}, \mathrm{CD} 41^{+}$, and $\mathrm{CD} 45^{+}$cells were sorted at different time points along EB differentiation by flow cytometer. Gene expression analyses were conducted with Agilent Whole Mouse Genome (014868) $4 \times 44$ multiplex format oligo arrays (Agilent technologies, USA) following the Agilent 1-color microarray-based gene expression analysis protocol. Starting with $500 \mathrm{ng}$ of the total RNA, Cy3 labeled cRNAs was produced according to manufacturer's protocol. For each sample, $1.65 \mu \mathrm{g}$ of Cy3 labeled cRNAs was fragmented and hybridized for $17 \mathrm{~h}$ in a rotating hybridization oven. Slides were washed and scanned with an Agilent Scanner. Data were obtained using the Agilent Feature Extraction software (v9.5), using the 1-color default for all parameters. The Agilent Feature Extraction software was also used for error modeling, adjusting for additive and multiplicative noises. The final data were processed using Rosetta Resolver system (v7.2, Rosetta Biosoftware, USA).

\section{Prediction of transcription factor binding sites and miRNA target genes}

Transcription factor binding sites at the promoters of miRNAs were predicted by the $\mathrm{R}$ programming language (https://www.bioconductor.org/) and the binding matrix was obtained from http://www.gene-regulation.com/pub/databases.html and http://jaspar.genereg.net/. The miRNA target genes and binding motifs were predicted via $\mathrm{http}$ ://www.targetscan.org.

Compliance and ethics The author(s) declare that they have no conflict of interest.

Acknowledgements The microarray work was performed at the sequence core facility of National Institute of Environmental Health Sciences (NIEHS). This study was supported by the Ministry of Science and Technology of China (2016YFA0100302, 2014CB964800), the National Natural Science Foundation of China (31471347, 30971522, 31271589), the Science and Technology Commission of Shanghai Municipality (11DZ2260300, 13JC1406402, 16JC1404200)

Bartel, D.P. (2004). MicroRNAs. Cell 116, 281-297.

Bertrand, J.Y., Giroux, S., Cumano, A., and Godin, I. (2005). Hematopoietic stem cell development during mouse embryogenesis. Methods Mol Med $105,273-288$.

Chao, R., Gong, X., Wang, L., Wang, P., and Wang, Y. (2015). CD71high population represents primitive erythroblasts derived from mouse embryonic stem cells. Stem Cell Res 14, 30-38.

Chen, C.Z., Li, L., Lodish, H.F., and Bartel, D.P. (2004). MicroRNAs modulate hematopoietic lineage differentiation. Science 303, 83-86.

Chen, D., and Zhang, G. (2001). Enforced expression of the GATA-3 transcription factor affects cell fate decisions in hematopoiesis. Exp Hematol $29,971-980$

Cumano, A., Dieterlen-Lièvre, F., and Godin, I. (2000). The splanchnopleura/AGM region is the prime site for the generation of multipotent hemopoietic precursors, in the mouse embryo. Vaccine 18, 1621-1623.

Dzierzak, E. (1999). Embryonic beginnings of definitive hematopoietic stem cells. Ann N Y Acad Sci 872, 256-264; discussion 262-254.

Elefanty, A.G., Robb, L., and Glenn Begley, C. (1997). Factors involved in leukaemogenesis and haemopoiesis. Baillieres Clin Haematol 10, 589-614.

Fatica, A., Rosa, A., Fazi, F., Ballarino, M., Morlando, M., De angelis, F.G., Caffarelli, E., Nervi, C., and Bozzoni, I. (2006). MicroRNAs and hematopoietic differentiation. Cold Spring Harb Symp Quant Biol 71, 205-210.

Fazi, F., Rosa, A., Fatica, A., Gelmetti, V., De Marchis, M.L., Nervi, C., and Bozzoni, I. (2005). A minicircuitry comprised of microRNA-223 and transcription factors NFI-A and C/EBPa regulates human granulopoiesis. Cell 123, 819-831.

Gangaraju, V.K., and Lin, H. (2009). MicroRNAs: key regulators of stem cells. Nat Rev Mol Cell Biol 10, 116-125.

Gao, X., Wu, T., Johnson, K.D., Lahvic, J.L., Ranheim, E.A., Zon, L.I., and Bresnick, E.H. (2016). GATA factor-G-protein-coupled receptor circuit suppresses hematopoiesis. Stem Cell Rep 6, 368-382.

Garzon, R., and Croce, C.M. (2008). MicroRNAs in normal and malignant hematopoiesis. Curr Opin Hematol 15, 352-358.

Godin, I., and Cumano, A. (2002). The hare and the tortoise: an embryonic haematopoietic race. Nat Rev Immunol 2, 593-604.

Gruber, A.J., Grandy, W.A., Balwierz, P.J., Dimitrova, Y.A., Pachkov, M., Ciaudo, C., Nimwegen, E., and Zavolan, M. (2014). Embryonic stem cell-specific microRNAs contribute to pluripotency by inhibiting 
regulators of multiple differentiation pathways. Nucleic Acids Res 42, 9313-9326.

Hermiston, M.L., Xu, Z., and Weiss, A. (2003). CD45: a critical regulator of signaling thresholds in immune cells. Annu Rev Immunol 21, 107-137.

Houbaviy, H.B., Murray, M.F., and Sharp, P.A. (2003). Embryonic stem cell-specific microRNAs. Dev Cell 5, 351-358.

Judson, R.L., Babiarz, J.E., Venere, M., and Blelloch, R. (2009). Embryonic stem cell-specific microRNAs promote induced pluripotency. Nat Biotechnol 27, 459-461.

Keller, G., Kennedy, M., Papayannopoulou, T., and Wiles, M.V. (1993). Hematopoietic commitment during embryonic stem cell differentiation in culture. Mol Cell Biol 13, 473-486.

Kennedy, M., D’Souza, S.L., Lynch-Kattman, M., Schwantz, S., and Keller, G. (2007). Development of the hemangioblast defines the onset of hematopoiesis in human ES cell differentiation cultures. Blood 109, 2679-2687.

Kennedy, M., Firpo, M., Choi, K., Wall, C., Robertson, S., Kabrun, N., and Keller, G. (1997). A common precursor for primitive erythropoiesis and definitive haematopoiesis. Nature 386, 488-493.

Kruse, E.A., Loughran, S.J., Baldwin, T.M., Josefsson, E.C., Ellis, S., Watson, D.K., Nurden, P., Metcalf, D., Hilton, D.J., Alexander, W.S., and Kile, B.T. (2009). Dual requirement for the ETS transcription factors Fli-1 and Erg in hematopoietic stem cells and the megakaryocyte lineage. Proc Natl Acad Sci USA 106, 13814-13819.

Kyba, M., and Daley, G. (2003). Hematopoiesis from embryonic stem cells: lessons from and for ontogeny. Exp Hematol 31, 994-1006.

Kyba, M., Perlingeiro, R.C.R., and Daley, G.Q. (2002). HoxB4 confers definitive lymphoid-myeloid engraftment potential on embryonic stem cell and yolk sac hematopoietic progenitors. Cell 109, 29-37.

Lensch, M.W., and Daley, G.Q. (2004). Origins of mammalian hematopoiesis: in vivo paradigms and in vitro models. Curr Top Dev Biol 60, 127-196.

Lohmann, F., and Bieker, J.J. (2008). Activation of Eklf expression during hematopoiesis by Gata2 and Smad5 prior to erythroid commitment. Development 135, 2071-2082.

Long, Y., and Huang, H. (2015). On signaling pathways: hematopoietic stem cell specification from hemogenic endothelium. Sci China Life Sci 58, 1256-1261.

Mankertz, J., Hillenbrand, B., Tavalali, S., Huber, O., Fromm, M., and Schulzke, J.D. (2004). Functional crosstalk between Wnt signaling and Cdx-related transcriptional activation in the regulation of the claudin-2 promoter activity. Biochem Biophys Res Commun 314, 1001-1007.

McKinney-Freeman, S.L., Lengerke, C., Jang, I.H., Schmitt, S., Wang, Y., Philitas, M., Shea, J., and Daley, G.Q. (2008). Modulation of murine embryonic stem cell-derived $\mathrm{CD} 41^{+} \mathrm{c}-\mathrm{kit}^{+}$hematopoietic progenitors by ectopic expression of $C d x$ genes. Blood 111, 4944-4953.

Medvinsky, A., and Dzierzak, E. (1996). Definitive hematopoiesis is autonomously initiated by the AGM region. Cell 86, 897-906.
Mikkola, H.K.A. (2003). Expression of CD41 marks the initiation of definitive hematopoiesis in the mouse embryo. Blood 101, 508-516.

Morrison, S.J., Uchida, N., and Weissman, I.L. (1995). The biology of hematopoietic stem cells. Annu Rev Cell Dev Biol 11, 35-71.

Nerlov, C., and Graf, T. (1998). PU.1 induces myeloid lineage commitment in multipotent hematopoietic progenitors. Genes Dev 12, 2403-2412.

Palis, J., Robertson, S., Kennedy, M., Wall, C., and Keller, G. (1999). Development of erythroid and myeloid progenitors in the yolk sac and embryo proper of the mouse. Development 126, 5073-5084.

Patrick, D.M., Zhang, C.C., Tao, Y., Yao, H., Qi, X., Schwartz, R.J., Jun-Shen Huang, L., and Olson, E.N. (2010). Defective erythroid differentiation in miR-451 mutant mice mediated by 14-3-3. Genes Dev 24, 1614-1619.

Porcher, C., Swat, W., Rockwell, K., Fujiwara, Y., Alt, F.W., and Orkin, S.H. (1996). The T cell leukemia oncoprotein SCL/tal-1 is essential for development of all hematopoietic lineages. Cell 86, 47-57.

Schmitt, R.M., Bruyns, E., and Snodgrass, H.R. (1991). Hematopoietic development of embryonic stem cells in vitro: cytokine and receptor gene expression. Genes Dev 5, 728-740.

Starck, J., Weiss-Gayet, M., Gonnet, C., Guyot, B., Vicat, J.M., and Morle, F. (2010). Inducible Fli-1 gene deletion in adult mice modifies several myeloid lineage commitment decisions and accelerates proliferation arrest and terminal erythrocytic differentiation. Blood 116, 4795-4805.

Vasilatou, D., Papageorgiou, S., Pappa, V., Papageorgiou, E., and Dervenoulas, J. (2010). The role of microRNAs in normal and malignant hematopoiesis. Eur J Haematol 84, 1-16.

Wang, Q., Liu, X., Tang, N., Archambeault, D.R., Li, J., Song, H., Tang, C., He, B., Matzuk, M.M., and Wang, Y. (2013). GASZ promotes germ cell derivation from embryonic stem cells. Stem Cell Res 11, 845-860.

Wang, Y., Baskerville, S., Shenoy, A., Babiarz, J.E., Baehner, L., and Blelloch, R. (2008). Embryonic stem cell-specific microRNAs regulate the G1-S transition and promote rapid proliferation. Nat Genet 40, 1478-1483.

Wang, Y., Yates, F., Naveiras, O., Ernst, P., and Daley, G.Q. (2005). Embryonic stem cell-derived hematopoietic stem cells. Proc Natl Acad Sci USA 102, 19081-19086.

Wilson, N.K., Foster, S.D., Wang, X., Knezevic, K., Schütte, J., Kaimakis, P., Chilarska, P.M., Kinston, S., Ouwehand, W.H., Dzierzak, E., Pimanda, J.E., de Bruijn, M.F.T.R., and Göttgens, B. (2010). Combinatorial transcriptional control in blood stem/progenitor cells: genome-wide analysis of ten major transcriptional regulators. Cell Stem Cell 7, 532-544.

Xie, J.J., and Zhang, C.C. (2015). Ex vivo expansion of hematopoietic stem cells. Sci China Life Sci 58, 839-853.

Xie, X.Y., Li, Y.H., and Pei, X.T. (2014). From stem cells to red blood cells: how far away from the clinical application? Sci China Life Sci $57,581-585$

Zhao, M., and Li, L.H. (2015). Regulation of hematopoietic stem cells in the niche. Sci China Life Sci 58, 1209-1215.

\section{SUPPORTING INFORMATION}

Table S1 Sequences of the oligonulceotides.

The supporting information is available online at life.scichina.com and www.springerlink.com. The supporting materials are published as submitted, without typesetting or editing. The responsibility for scientific accuracy and content remains entirely with the authors. 